\title{
Pour une transformation des épreuves classantes nationales en un examen classant national
}

\author{
Shifting from national ranking examinations \\ to a national ranking assessment
}

\begin{abstract}
Résumé Propos : Les Epreuves classantes nationales (ECN) sont organisées à l'issue du deuxième cycle des études médicales en France. Elles ne constituent ni un examen - puisqu'aucune performance minimale n'est requise -, ni un concours - puisque le nombre des postes offerts dépasse le nombre de candidats -. Tous les étudiants qui se présentent sont admis en rang utile pour choisir un poste d'interne. Exégèse : L'auteur plaide pour une évolution de simples épreuves nationales classantes vers un examen national classant, qui aurait une fonction certificative en fixant un niveau minimal de performance à satisfaire. Il explore les enjeux et la faisabilité d'une telle évolution. Conclusions : La décision de transformer les épreuves classantes nationales de fin de second cycle en examen national classant et certificatif comporte une dimension de nature à la fois pédagogique et politique. Elle clarifierait les objectifs à satisfaire pour les étudiants et réorienteraient favorablement l'activité des examinateurs au profit de la pertinence de l'examen.
\end{abstract}

Abstract Purpose: In France, National ranking examinations (NRE) are organized at the end of the second cycle of medical studies (6th year). They are neither an exam, as no minimal level is required, nor a competitive examination, as the number of positions overpasses the number of candidates. All students taking NRE are allowed to choose a position of resident. Exegesis: The author pleads for an evolution from simple national ranking examinations to a national ranking assessment, that would have a certificating function to ascertain a suitable level of performance. He explores the stakes and the feasibility of such an evolution. Conclusions: The decision to shift from simple national ranking examinations at the end of the second cycle to a national ranking assessment, has pedagogical and political dimensions. It would clarify the objectives to fulfil for students and it would positively transform the examinators' activity towards the relevance of the exam.

Pédagogie Médicale 2006; 7: 228-32

\footnotetext{
* Francis Roussel - Département d'Anatomie et de Cytologie Pathologiques - Faculté de Médecine - 76000 Rouen - France. Correspondance : Francis Roussel - Pavillon J Delarue - CHU de Rouen - 76031 Rouen Cedex. Tel : + 33 (0)2 32888212 Mailto:Francis.Roussel@univ-rouen.frou Francis.Roussel@chu-rouen.fr
} 


\section{"Décidément les Français sont gens fort extravagants " ${ }^{1}$ Montesquieu}

Seul le Persan fraîchement débarqué ${ }^{1}$, croyant de bonne foi que, depuis trois ans, le second cycle des études médicales en France est sanctionné par un examen classant national, pourrait s'interroger sur le titre de cette tribune. En réalité, à l'issue de la fin de ce second cycle, c'est-à-dire à la fin des six années d'études qui constituent en France le cursus " prégradué " de la formation médicale initiale, c'est bien à l'aune de leurs résultats à des épreuves classantes nationales $(\mathrm{ECN})^{2}$ que les étudiants français sont contraints de se mesurer s'ils veulent accéder, selon leur " mérite " c'est-à-dire à l'issue d'un choix effectué selon leur rang de classement, à l'une ou l'autre des filières de formation spécialisées du troisième cycle (cursus "postgradué ») et exercer dans l'un des centres hospitalo-universitaires français les fonctions correspondantes d'interne. En effet, selon une terminologie largement partagée ${ }^{3}$, un examen fait l'objet d'une interprétation normative en fondant son verdict (reçu ou refusé) sur le critère d'un score minimal ; dans le cas d'un concours, l'interprétation normative prend en compte le rang de classement des étudiants et vise la sélection d'un nombre prédéterminé de candidats. Dans le cas des ECN, il s'agit de classer et seulement de classer les candidats. Les ECN ont donc cette particularité remarquable de ne générer "ni refusé, ni reçu ". Elles ne sont ni un examen, ni un concours.

Nous plaidons en faveur d'une transformation de ces épreuves classantes en examen classant en arguant qu'il s'agit d'une décision de nature à la fois pédagogique et politique. En tant que décision de nature pédagogique, il s'agit d'une part, d'optimiser le caractère discriminant des épreuves en rendant plus tangibles les écarts de scores entre les candidats; et d'autre part, de convenir que, si leurs performances à l'examen sont insuffisantes, certains étudiants seront refusés. En tant que décision politique, dans le contexte spécifique "francofrançais ", il s'agit d'admettre que la qualité métrologique des épreuves n'est pas à la hauteur de l'équité républicaine qu'elles sont pourtant censées satisfaire pour départager des citoyens candidats qui, de fait, jouent l'orientation de toute leur carrière sur des différences de scores infimes obtenues à une unique épreuve.

Un des inconvénients des ECN telles qu'elles sont actuellement organisées est de permettre le passage en troisième cycle d'étudiants faibles, voire très faibles.
A titre indicatif, l'étudiant classé en dernier rang lors de la session 2005 n'avait obtenu que 121 des 900 points possibles. Peu d'instituteurs de deuxième année de cours moyen (CM2) - dernière année du cursus à l'école primaire en France - laisseraient passer en classe de sixième - première année du cursus secondaire - un élève ayant 2,5/20 de moyenne et l'on comprend mal ce constat de "deux poids - deux mesures" : pourquoi postule-t-on, d'un côté, que l'intérêt bien compris d'un apprenant de classe CM2 en difficulté est de combler d'abord ses lacunes avant de passer au niveau supérieur, alors que, de l'autre, on considère que cela n'est pas nécessaire pour le futur interne. En outre, il est coutumier d'entendre les examinateurs se plaindre de l'impossibilité de " coller " un candidat qui aurait " tué son malade ». Cette remarque de bon sens est un autre argument fort, en faveur de la transformation des épreuves en examen. Il devrait d'ailleurs être possible d'effectuer des cotations négatives fortes (supérieures aux quatre points seulement qu'autorisent les actuelles dispositions réglementaires ${ }^{2}$ ) ou de déterminer que des scores inférieurs à un certain seuil auraient un caractère éliminatoire ${ }^{4}$. Bien sûr, on peut objecter qu'en principe, il a été vérifié au sein de chacune des facultés, à l'aide des épreuves obligatoires du certificat de synthèse clinique et thérapeutique (CSCT), que tous les étudiants admis à se présenter à l'ECN avaient un niveau suffisant ; certains étudiants sont ainsi refusés dans leur faculté à l'examen de fin de dernière année de deuxième cycle et doivent redoubler cette année d'études ; ceci pourrait dès lors légitimer la seule fonction classante des ECN. Cependant, les exigences des facultés sont variables et quelquefois entachées d'arrière-pensées stratégiques visant à rendre plus flatteur leur rang au palmarès annuel officieux des facultés qui est élaboré à partir des résultats des étudiants aux $\mathrm{ECN}$. Le caractère national des épreuves constitue donc un progrès structurel. De même, l'introduction d'épreuves d'analyse de dossiers cliniques est un progrès pédagogique sensible par rapport au psittacisme ancestral que favorisaient les anciennes épreuves qui, avec des questions énoncées le plus souvent sous la forme " signes, diagnostic, traitement » ou encore " conduite à tenir devant... ", n'exploraient que le seul rappel de connaissances déclaratives

Il n'est pas question de revenir sur ces points, bien qu'il existe un fort courant de pensée pour plaider la suppression de l'épreuve nationale ou pour sa régionalisation, ce qui revient au même. L'épreuve est accusée d'être le moteur de transhumances estudiantines aboutissant à la catastrophe de l'année 2005 concernant le déficit de 
recrutement de près de 800 internes en médecine générale ${ }^{5}$. Cette interprétation est fausse (figure 1). Chez les futurs spécialistes, pour qui la tradition de mobilité fréquente dans le cadre de l'ancien concours d'internat est séculaire, on observe encore une augmentation du taux de changement de région entre 2004 et 2005. Le déterminant de cette mobilité est donc d'une autre nature. Chez les futurs généralistes, l'évolution entre 2004 et 2005 se produit dans le même sens mais est beaucoup plus ample traduisant un rattrapage. Il s'agit donc bien d'un fait de société, de l'adaptation à une mobilité dépassant le monde de la médecine. Et par ailleurs, même si le nombre de places d'internes offertes n'avait pas largement excédé le nombre d'étudiants à répartir, les migrations n'auraient eu aucune influence sur la saturation des postes. En revanche, l'option de régionaliser les ECN n'est pas neutre : elle revient à imposer une contrainte géographique à l'affectation de la totalité des internes et donc à fixer dans leur région de second cycle les étudiants, ou plus encore, les seuls futurs généralistes. Or, les étudiants de premier et de second cycle sont déjà contraints de s'inscrire dans la faculté de leur région d'origine et les différences de taux d'encadrement appréciés par le ratio enseignants/étudiants entre établissements sont majeures (cœfficient multiplicatif de 2,25 entre les facultés respectivement les plus pauvrement et les plus richement dotées). Les étudiants sont ainsi placés dans des situations très diverses qui constituent actuellement un des déterminants de la réussite aux $\mathrm{ECN}^{5}$. L'iniquité d'une telle situation est flagrante. Sa pérennisation au siècle des bourses Erasmus - qui visent à favoriser les échanges internationaux européens des étudiants au cours des études - ne va pas de soi. L'ouverture des frontières permet en outre à des médecins de niveau très variable de s'installer partout. Il est facile aux enseignants et aux médecins de dire que le niveau de ceux qui viennent s'installer en France doit être au-dessus d'un minimum. Encore faudrait-il que les dispositions en vigueur pour les étudiants formés en France exigent d'eux qu'ils démontrent avoir atteint un niveau minimum explicite. La transformation des ECN en examen national classant offre cette possibilité. Sa diffusion permettrait de réguler l'espace européen de mobilité professionnelle.

Techniquement, la décision de fixer un seuil minimal pour la note de passage demande la modification d'un arrêté $^{2}$ et c'est bien en cela que la question a un caractère politique. L'article 7 pourrait, par exemple, préciser que " la note de passage est fixée par le jury » ou indiquer des procédures pour la déterminer ; celles-ci pourraient être univoques et fixes ou au contraire évolutives car la position définitive de la barre pourrait être définie par étapes. En effet, si la définition d'une note de passage est un point délicat, l'introduction de son usage en est un autre. Elle doit être acceptable, car elle influencera la quantité et la qualité du travail fourni par les étudiants, en particulier par les plus faibles. De nombreuses modélisations peuvent être faites. Huit d'entre elles sont indiquées (Tableau 1). Par exemple, dans les conditions des épreuves de 2005 , le fait de placer la barre à 300 points sur 900 - c'est-à-dire décider que la formulation d'une réponse fausse à plus de deux items sur trois était éliminatoire - aurait théoriquement entraîné le redoublement de 64 étudiants sur 4232 . Un réglage graduel de la hauteur de la barre pourrait être fait sur trois ou quatre sessions.

Les caractéristiques docimologiques des ECN en seraient radicalement transformées. Il est remarquable que le meilleur score obtenu en 2005 a été de 711 sur 900. En d'autres termes, l'étudiante majore de la promotion n'avait pas formulé une réponse correcte pour $21 \%$ des items pris en compte pour la correction. On peut penser qu'une partie au moins de ces items étaient trop spécialisés et finalement inadaptés à ce stade de la formation des étudiants; dès lors, il serait sans doute judicieux d'évoluer vers des items testant un savoir plus indispensable. La distribution des notes s'étalerait et on cesserait de voir les scores finaux des 32 étudiants "dilués » dans la moitié de l'espace de notation et ceux des 3400 restants concentrés dans l'autre moitié. Il conviendrait d'être rigoureux pour garantir la précision de la mesure des performances des étudiants dont les scores sont proches de la limite, quitte à recourir pour cela à d'autres modalités d'examen ${ }^{7}$. L'activité pédagogique des enseignants se déplacerait avec profit vers la conception des questions plutôt que vers le contrôle stérile des réponses.

Il est en notre pouvoir, il est de notre devoir, que l'étonnement du Persan, génialement décrit en 1721, cesse d'être d'actualité dans le domaine de la formation des médecins. 
Figure 1 :

Mobilité par grande filière : pourcentage d'étudiants choisissant une région de troisième cycle différent de leur région d'origine
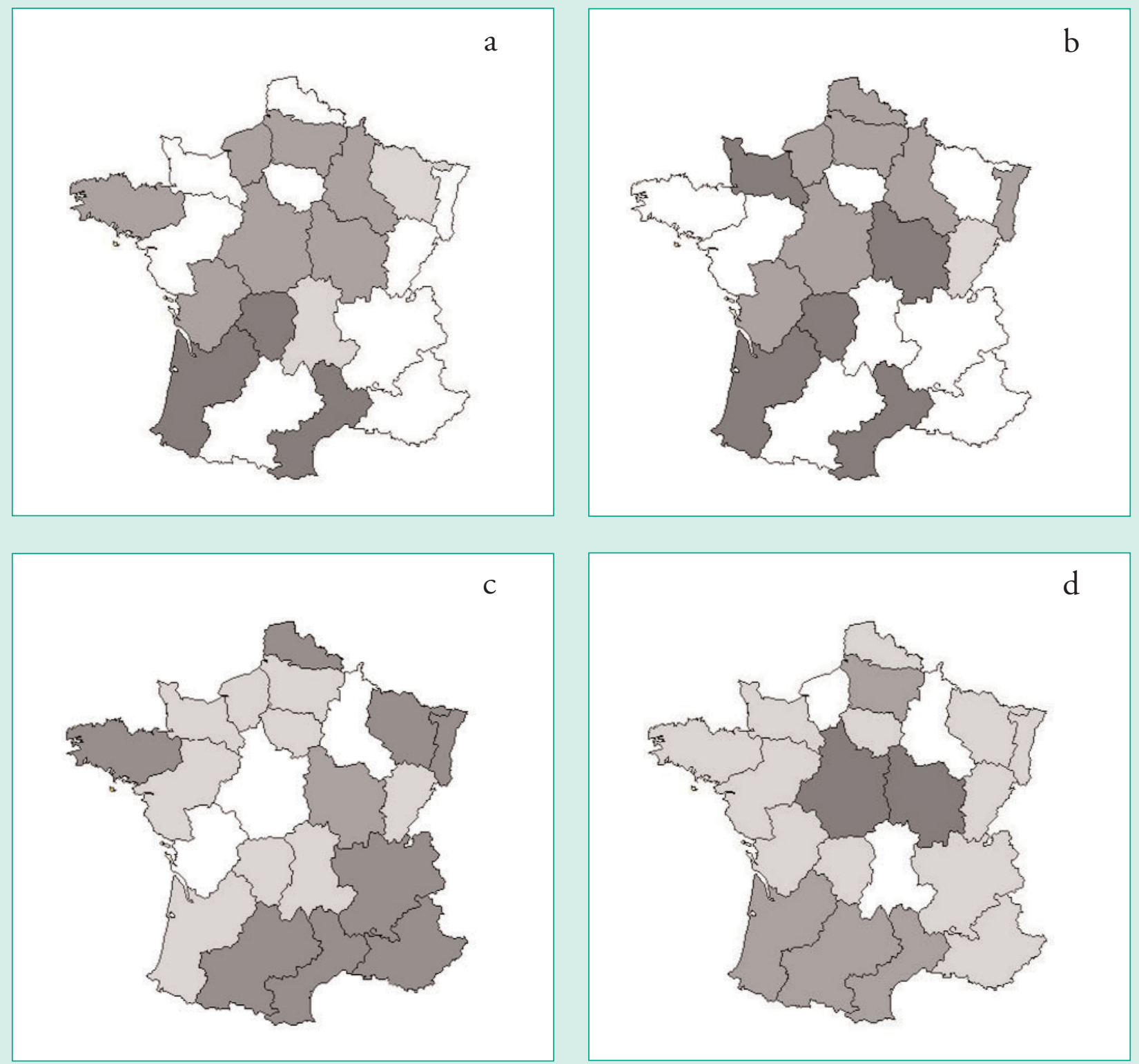

Légende
$23,52 \%$ à $37,45 \%$
$37,45 \%$ à $51,37 \%$
$51,37 \%$ à $65,30 \%$
$65,30 \%$ à $79,23 \%$
$79,23 \%$ à $93,15 \%$

( $a, b$ : futurs spécialistes ; $c, d$ : futurs généralistes ; $a, c: 2004 ; b, d: 2005$ ) 


\section{Tribune}

\begin{tabular}{|c|c|c|c|c|}
\hline \multicolumn{7}{|c|}{$\begin{array}{c}\text { Tableau 1 : } \\
\text { Effet théorique de différents seuils de réussite appliqués } \\
\text { aux résultats de la promotion } 2005\end{array}$} \\
\hline $\begin{array}{c}\text { Seuil exprimé } \\
\text { en score/900 }\end{array}$ & $\begin{array}{c}\text { Seuil exprimé } \\
\text { en score/20 }\end{array}$ & $\begin{array}{c}\text { Score seuil/distribution } \\
\text { (moyenne-écart-type) }\end{array}$ & $\begin{array}{c}\text { nombre d'étudiants } \\
\text { refusés }\end{array}$ & $\begin{array}{c}\text { \% d'étudiants } \\
\text { refusés }\end{array}$ \\
\hline 372,5 & 8,28 & 2 & 177 & 4,18 \\
\hline 337,5 & 7,5 & 2,44 & 101 & 2,39 \\
\hline 332,5 & 7,39 & 2,5 & 94 & 2,22 \\
\hline 315 & 7 & 2,73 & 76 & 1,80 \\
\hline 300 & 6,66 & 2,91 & 64 & 1,51 \\
\hline 292,5 & 6,5 & 3 & 60 & 1,42 \\
\hline 270 & 6 & 3,28 & 41 & 0,97 \\
\hline 225 & 5 & 3,84 & 20 & 0,47 \\
\hline
\end{tabular}

\section{Références}

1. Montesquieu. Lettres Persanes. Paris : Alphonse Lemerre Ed. 1873.

2. Arrêté du 29 janvier 2004 relatif à l'organisation des épreuves classantes nationales anonymes donnant accès au troisième cycle spécialisé des études médicales. Journal officiel de la République Française. [On-line]. Disponible sur:http://www.legifrance.gouv.fr/WAspad/UnTexteDeJorf NOR: SANH0420335A

3. Legendre $R$. Dictionnaire actuel de l'éducation. Montréal : Guérin et Paris : Eska (2e édition), 2000.

4. Le Bourgeois JP. Epreuves classantes nationales, pourquoi et comment... Presse Med. 2005;34:767-8.
5. Roussel F. Epreuves classantes nationales, une inadéquation majeure entre le nombre des postes. Presse Med. 2006 ;35:437-8.

6. Roussel F, Gehanno J-F, Ladner J, Benichou J. Do teaching faculty resources affect the choice of medical career? Sous presse in : Medical Teacher.

7. Fournier JP, De Champlain AF, Benchimol D, Staccini P, Subhiyah $R$, Braun $M$ et al. Intérêt de transposer un examen américain dans le cadre $d u$ futur examen national classant validant français. Étude préliminaire. Ann Med Interne (Paris). 2003; 154:148-56. 